\title{
Mycobacterium tuberculosis: 2014 Clinical trials in review
}

\author{
Kevin B Laupland MD MSc FRCPC ${ }^{1,2}$, Louis Valiquette MD MSc FRCPC 3
}

$\mathrm{T}$ uberculosis (TB) is a major cause of death and suffering worldwide. Based on data from the WHO, an estimated nine million new cases were diagnosed in 2013, with 1.5 million associated deaths (1.1 million HIV-negative cases and 0.36 million HIV-positive cases) (1). Worldwide incidence and mortality rates have been declining over the past decade due to enhanced diagnosis and management of both TB and HIV infection. However, multidrug-resistant TB, which occurs in approximately $4 \%$ of cases, is challenging these advances (2). The past year witnessed the publication of several clinical trials surrounding TB management and control. In the present note, we briefly review and highlight clinical trials from the past year evaluating new diagnostic and therapeutic strategies for managing TB in adults.

\section{DIAGNOSTICS}

Traditionally, the diagnosis of active TB has been based on presumptive diagnosis using microscopy with culture confirmation, and the diagnosis of latent TB infection using skin testing and/or interferongamma release assay (IGRA). Several clinical trials evaluating different or new approaches to TB diagnosis were reported in 2014.

\section{Basic versus enhanced TB diagnostics}

Reddy et al (3) randomly assigned 70 patients in Tanzania to management based on smear results alone versus concentrated smear and culture results. They observed that appropriate treatment was higher among patients managed using the more intensive laboratory regimen. However, this study was limited by small size, a high mortality rate in both groups and difficulties in establishing appropriateness of care in cases for which cultures were not performed.

\section{Laboratory information systems}

Blaya et al (4) conducted a cluster-randomized trial among 78 primary health centres in Lima, Peru, to evaluate the use of an Internet-based laboratory information system. They found that the times to receive culture and drug susceptibility test results, as well as the time to culture conversion, were significantly reduced by use of the system. This study is important in that it demonstrates the value of using novel means to communicate information regarding improvements in TB care delivery.

\section{IGRAs}

Due to the many limitations of standard TB skin testing, IGRAs have been increasingly used for the diagnosis and management of active or latent TB. Johnson et al (5) investigated the use of serial QuantiFERON-TB Gold In-Tube tests (Cellestis Ltd, Australia) as a potential biomarker of response to latent TB therapy with isoniazid in HIV-negative South African adults. However, no significant differences were observed.

The value of IGRA testing to direct TB therapy among HIV-positive individuals is controversial. Danel et al (6) reported on a substudy of the Temprano trial to assess the utility of using the QuantiFERON-TB Gold In-Tube test to diagnose TB in HIV-positive individuals who did not have low CD4 cell counts in Cote d'Ivoire. They found that this test had a sensitivity of $88 \%$, a specificity of $66.6 \%$, and positive and negative predictive values of $6.5 \%$ and $99.5 \%$, respectively. These data suggest that this test, when negative, may be used to rule out TB in HIV-positive adults who are not severely immunosuppressed with a high degree of certainty. However, in another trial conducted by Rangaka et al (7) from South Africa, HIV-positive patients without evidence of active TB were randomly assigned to receive antiretroviral therapy plus either isoniazid or placebo. They observed that, among those testing negative using QuantiFERON-TB Gold In-Tube test at baseline, that TB rates were subsequently 3 per 100 person-years of observation and 1.3 per 100 personyears of observation in the placebo and isoniazid groups, respectively. The inability to rule out latent TB in these patients using the IGRA may have been confounded by the degree of immune suppression.

\begin{abstract}
Molecular diagnostics
Molecular diagnostics have been increasingly applied to improve the time to diagnosis of TB and to identify drug-resistant strains. Durovni et al (8) conducted a cluster-randomized trial in 14 laboratories in Brazil that compared smear microscopy and the polymerase chain reaction-based Xpert MTB/RIF assay (Cepheid, USA). They found that compared with smear microscopy, the Xpert MTB/RIF assay identified more positive cases (14.2\% versus $9.7 \%$ ), and this was associated with earlier time to treatment. In another study conducted in primary care settings in southern Africa, Theron et al (9) evaluated nurseperformed point-of-care Xpert MTB/RIF compared with smear microcopy. They found that point-of-care Xpert MTB/RIF testing was of higher sensitivity than smear ( $83 \%$ versus $50 \%$, respectively) and that field-performed Xpert MTB/RIF testing was as sensitive and more specific than subsequent laboratory-performed Xpert MTB/RIF testing. These data indicate that molecular tests are feasible and of high utility in the field setting, and may lead to earlier treatment prescription (9).
\end{abstract}

\section{CHEMOTHERAPY}

New agents and chemotherapeutic strategies

Anti-TB chemotherapy is the mainstay of TB therapy. Several clinical trials reported over the past year evaluated new agents or strategies for TB treatment; these are summarized in Table 1 (10-14).

\section{HIV coinfection}

Patients with HIV are at increased risk for acquiring and developing severe disease due to TB. An important body of literature continues to evolve surrounding the management of this important coinfection (15).

\begin{abstract}
Timing of initiation of antiretroviral therapy
Grinsztejn et al (16) conducted a multicentre trial comparing early (at study entry) versus delayed (onset of AIDS-relayed illness or declining CD4 count to approximately 200 cells $/ \mu \mathrm{L}$ ) initiation of antiretroviral treatment on several outcomes including the development of TB. Among the 1763 individuals enrolled, TB developed in
\end{abstract}

${ }^{1}$ Department of Medicine, Royal Inland Hospital, Kamloops, British Columbia; ${ }^{2}$ Departments of Medicine, Critical Care Medicine, Pathology and

Laboratory Medicine, and Community Health Sciences, University of Calgary, Calgary, Alberta; ${ }^{3}$ Department of Microbiology-Infectious

Diseases, Université de Sherbrooke, Sherbrooke, Quebec

Correspondence: Dr Kevin B Laupland, Royal Inland Hospital, 311 Columbia Street, Kamloops, British Columbia V2C 2T1.

Telephone 250-374-5111, e-mail klaupland@gmail.com

\footnotetext{
OPEN ACCESS

This open-access article is distributed under the terms of the Creative Commons Attribution Non-Commercial License (CC BY-NC) (http:// creativecommons.org/licenses/by-nc/4.0/), which permits reuse, distribution and reproduction of the article, provided that the original work is properly cited and the reuse is restricted to noncommercial purposes. For commercial reuse, contact support@pulsus.com
} 
TABLE 1

An overview of studies published in 2014 describing new agents/strategies for treating tuberculosis

\begin{tabular}{llll}
\hline Author (reference) Population & Comparison & Results \\
\hline Merle et al (10) & $\begin{array}{l}\text { Smear positive, } \\
\text { RIF sensitive }\end{array}$ & $\begin{array}{l}\text { RIF and INH with either ETH for six months or } \\
\text { GAT for four months }\end{array}$ & $\begin{array}{l}3.5 \% \text { higher rate of unfavourable } \\
\text { outcome with four-month regimen } \\
\text { largely related to higher rate of } \\
\text { recurrence }\end{array}$
\end{tabular}

Jindani et al (11) Smear positive drug sensitive

Dorman et al (12) Smear positive

Gillespie et al (13) Smear positive

Diacon et al (14) Smear positive, multidrug-resistant tuberculosis
Standard regimen (ETH and PYR for two months Unfavourable outcomes in modified The six-month MOX and RPT plus RIF and INH for six months); two-month intention-to-treat analysis: $14.4 \%$ regimen (MOX replaces INH in control regimen in the standard regimen, $13.7 \%$ for two months, then MOX plus RPT for two in the six-month regimen and months); six-month regimen (MOX replaces $\quad 26.9 \%$ in the four-month regimen $\mathrm{INH}$ in control regimen for two months then MOX plus RIF weekly for four months) INH, PYR and ETH plus RIF OR $10 \mathrm{mg} / \mathrm{kg}$, $15 \mathrm{mg} / \mathrm{kg}$ or $20 \mathrm{mg} / \mathrm{kg} \mathrm{RPT}$

\section{Liquid cultures at eight weeks negative in $56 \%$ (RIF) versus $75 \%, 70 \%$ and $83 \%$ for $10 \mathrm{mg} / \mathrm{kg}$, $15 \mathrm{mg} / \mathrm{kg}$ and $20 \mathrm{mg} / \mathrm{kg}$ RPT groups, respectively}

Standard regimen (ETH and PYR for two months Time to culture negativity shortened Noninferiority of the plus RIF and INH for 18 weeks); standard regimen with $\mathrm{MOX}$ replacing $\mathrm{ETH}$ for 17 weeks; standard regimen with MOX replacing INH for 17 weeks

Backround regimen plus either $400 \mathrm{mg}$ of bedaquiline once daily for two weeks followed by $200 \mathrm{mg}$ three times per week for 22 weeks, or placebo in the MOX-containing groups, but these were associated with lower rates of favourable outcome

Median time to culture conversion 125 days for placebo compared with 83 days for bedaquiline, but mortality rates of $2 \%$ versus $13 \%$, respectively regimen was comparable with the standard regimen four-month regimen not shown
High-dose RPT associated negativity at eight weeks

\section{Addition of bedaquiline} resulted in faster culture conversion. Possibility of higher mortality rate requires further investigation with higher sputum culture

ETH Ethambutol; GAT Gatifloxacin; INH Isoniazid; MOX Moxifloxacin; PYR Pyrazinamide; RIF Rifampin; RPT Rifapentine

17 of 886 versus 34 of 875 patients, respectively (HR 0.49 [95\% CI 0.28 to 0.89 ]; $\mathrm{P}=0.018$ ).

Several previous studies have demonstrated a benefit of early initiation of antiretroviral therapy among HIV-positive patients with TB (17-22). Padayatchi et al (23) conducted a subanalysis of the SAPiT trial and evaluated outcomes of patients with multidrugresistant TB among those randomly assigned to initiation of antiretroviral therapy within 12 weeks of starting versus initiation after completion of TB therapy. Although the number of patients was small, the authors found an $86 \%$ reduction in mortality due to early initiation of antiretroviral therapy, which was statistically significant.

In response to questions surrounding the effect of early antiretroviral therapy influences outcome among HIV-TB coinfected patients who did not have low CD4 cell counts, Mfinanga et al (24) conducted a randomized trial comparing early (two weeks after starting TB therapy) versus delayed (after six months of TB therapy) antiretroviral treatment among 1675 African patients with HIV and CD4 cell counts $\geq 220$ cells $/ \mu \mathrm{L}$. They did not find a significant difference in outcome associated with early versus delayed treatment in this cohort.

While earlier antiretroviral therapy improves immune function and reduces the incidence and mortality associated with TB, immune reconstitution inflammatory syndrome is a potentially serious complication. Luetkemeyer et al (25) reported on a secondary analysis of the STRIDE clinical trial that evaluated the effects of earlier (two weeks of TB therapy) compared with later (eight to 12 weeks after TB treatment) antiretroviral therapy among HIV-positive patients with TB stratified according to CD4 cell counts ( $<50$ cells/ $\mu \mathrm{L}$ versus $\geq 50$ cells $\mu \mathrm{L})$ (19). They observed a rate of TB immune reconstitution inflammatory syndrome of $7.6 \%$ overall, but the rate was $44.3 \%$ among patients with early antiretroviral therapy and CD4 cell counts of $<50$ cells $/ \mu L(25)$.

Antiretroviral and TB chemotherapy optimization

Multiple drug combinations are needed for TB and HIV therapies, and drug interactions and toxicities present a challenge during comanagement of these infections. Because of limited interactions with rifampin, efavirenz is commonly used in antiretroviral regimens among patients with HIV-TB coinfection. However, intolerance or resistance to this drug is limiting for many patients. Grinszteyn et al (26) conducted a randomized trial comparing tenofovir/lamivudine with either two doses of raltegravir (400 mg and $800 \mathrm{mg}$ twice per day) or efavirenz (600 mg/day) among patients with HIV-TB coinfection. They found that the adverse event profile was similar and rates of virological suppression (raltegravir $400 \mathrm{mg}, 76 \%$; raltegravir $800 \mathrm{mg}, 78 \%$; and efavirenz, 63\%) at 48 weeks of follow-up were similar, indicating that raltegravir is an option for treatment of HIV-TB coinfected patients.

The rifampin-protease inhibitor interactions limit the use of these agents in HIV-TB coinfected patients. Because it has fewer interactions, rifabutin has been proposed as an alternative to rifampin. Lan et al (27) conducted a randomized crossover pharmacokinetic trial comparing two doses of rifabutin (150 mg three times per week versus $150 \mathrm{mg}$ daily) along with lopinavir/ritonavir. Among the 24 patients included, they observed that dosing of $150 \mathrm{mg}$ daily is preferred when coadministered with lopinavir/ritonavir. In another randomized crossover pharmacokinetic trial, Naiker et al (28) found that a daily dose of $150 \mathrm{mg}$ of rifabutin with lopinavir/ritonavir therapy resulted in adequate drug levels compared with $300 \mathrm{mg}$ daily, and was superior to $150 \mathrm{mg}$ three times weekly.

\section{VACCINES AND IMMUNOTHERAPY}

While chemotherapy is the mainstay of TB management, prevention using vaccines or therapy with immune adjuvants are important potential options that have been evaluated using clinical trials in 2014.

\section{Bacille Calmette-Guerin}

Hatherill et al (29) conducted a randomized trial evaluating the safety and reactogenicity of Bacille Calmette-Guerin (BCG) vaccination among $82 \mathrm{HIV}$-negative adult patients with latent TB infection who were immunized with BCG in infancy. Patients were given six months of isoniazid either before or after intradermal revaccination with BCG. While injection site erythema, induration and ulceration were 
common, no serious complications were observed. Pretherapy with isoniazid did not influence reactogenicity. This study indicates that BCG revaccination is safe among patients with latent $T B$ and provides important data to inform for future TB vaccine trials.

\section{Novel vaccines}

Several potential vaccine candidates were evaluated for safety and immunogenicity in phase I/II trials. Thacher et al (30) evaluated the candidate vaccine M72/AS01 by randomly assigning $37 \mathrm{HIV-infected}$ patients on antiretroviral therapy in a 3:1:1 ratio to vaccine, adjuvant (AS01) and saline placebo. The vaccine was clinically well tolerated and immunogenic. Reither et al (31) investigated the candidate vaccine $\mathrm{H} 1 / \mathrm{IC} 31$ in 48 HIV-positive patients and found that the vaccine was associated with a durable immune response. Nell et al (32) evaluated the RUTI vaccine (three doses and placebo) in 95 patients with latent TB infection. They observed a high rate of local injection site reactions, but the vaccine was immunogenic. These studies lay important groundwork for future trials aimed at improving clinical outcomes.

\section{Adjunctive, nutritional and other supportive therapies}

The role of adjuvant corticosteroid therapy in patients with pericardial TB has been uncertain. In addition, immunotherapy with heat-killed Mycobacterium indicus pranii has been proposed to improve outcomes in HIV-positive patients with TB. Mayosi et al (33) randomly assigned 1400 patients with TB pericardial disease in a $2 \times 2$ factorial design to receive steroid therapy or placebo, or heat-killed $M$ indicus pranii injections or placebo. While corticosteroid therapy was associated with a reduction in complications, there were no mortality benefits observed with either therapy overall. Both therapies were associated with a small but significant increased risk for cancer in HIV-positive patients. This study fails to show a significant overall benefit to use of adjunctive corticosteroid therapy or immunotherapy with $\mathrm{M}$ indicus pranii on the outcomes of patients treated for TB pericarditis.

TB chemotherapy is not uncommonly complicated by drugassociated hepatotoxicity, and a number of supplements have been proposed to reduce these adverse effects. Tabarsi et al (34) conducted a trial comparing daily $1000 \mathrm{mg}$ garlic tablet supplementation versus placebo during the first two weeks of TB therapy among 58 patients with pulmonary TB. They observed an overall rate of hepatotoxicity of $13 \%$ with no difference in the treatment groups overall. Hatamkhani et al (35) evaluated the effect of oral carnitine supplementation versus placebo on the occurrence of hepatotoxicity associated with TB chemotherapy. Although the rate of loss to follow-up was higher in the treatment group, therapy with oral carnitine was associated with a lower risk for TB therapy-associated transaminitis.

Chand et al (36) evaluated the effect of adjunctive homeopathic medicine versus placebo in the management of patients treated for multidrug-resistant TB among 120 patients in India. Other than an

\section{REFERENCES}

1. World Health Organization Global Health Observatory (GHO). How many TB cases and deaths are there? < www.who.int/gho/tb/ epidemic/cases_deaths/en/> (Accessed January 7, 2015).

2. World Health Organization, Global Tuberculosis Report 2014. $<$ http://apps.who.int/iris/bitstream/10665/137094/1/9789241564809_ eng.pdf $>$ (Accessed January 7, 2015).

3. Reddy EA, Njau BN, Morpeth SC, et al. A randomized controlled trial of standard versus intensified tuberculosis diagnostics on treatment decisions by physicians in Northern Tanzania. BMC Infect Dis 2014;14:89.

4. Blaya JA, Shin SS, Yagui M, et al. Reducing communication delays and improving quality of care with a tuberculosis laboratory information system in resource poor environments: A cluster randomized controlled trial. PLoS One 2014;9:e90110.

5. Johnson JL, Geldenhuys H, Thiel BA, et al. Effect of isoniazid therapy for latent TB infection on QuantiFERON-TB gold in-tube responses in adults with positive tuberculin skin test results in a high TB incidence area: A controlled study. Chest 2014;145:612-7. improvement in chest radiography associated with homeopathic treatment, no significant differences were observed among the multiple other outcomes evaluated.

The presence of HIV infection and malnutrition has been shown to influence the pharmacokinetics of rifampin. Jeremiah et al (37) conducted a clinical trial among 100 patients with pulmonary TB and randomly assigned them to receive either nutritional supplementation with high-energy vitamin- and mineral-fortified biscuits or nothing during the first two months of TB treatment. They observed that while rifampin concentrations were not different among HIV-negative patients, those that were HIV-positive and received nutritional supplementation had higher rifampin concentrations. These data suggest that nutritional supplementation may improve the success of TB therapy among HIV-positive patients in low-income countries.

\section{MULTIDISCIPLINARY AND PROGRAMMATIC INTERVENTIONS}

Identifying cases and delivery of therapy is the mainstay of TB management. However, in resource-limited jurisdictions, it is not clear to what degree additional expenditures on case finding and management may have on outcomes. Yadav et al (38) performed a clusterrandomized trial in 30 health districts in Cambodia and compared an extensive active case-finding program with usual passive case-finding activities. They observed a mortality reduction with active case finding of $2 \%$ compared with $14 \%$ for passive case finding, and that the active case finding was associated with a cost effectiveness of $\$ 330$ per disability-adjusted life-years or $\$ 5,300$ per death averted.

Churchyard et al (39) conducted a large (nearly 80,000 subjects) cluster-randomized trial among South African miners and compared a strategy of active screening and treatment of patients with active or latent TB versus usual care. The primary outcome was the incidence of TB 12 months after the intervention phase. While they observed decreased incidence of $\mathrm{TB}$ during the intervention phase, there were no differences in TB incidence after 12 months.

\section{SUMMARY AND CONCLUSIONS}

The past year witnessed the publication of a number of important clinical trials surrounding TB management. These have ranged from small phase 1 and 2 studies investigating novel vaccines and therapies to large population-level cluster-randomized studies investigating complex diagnostic and therapeutic strategies. We recognize that this note provides only a superficial summary of these studies and we likely failed to include some important trials that were published in 2014. Our intention was to highlight the breadth and importance of clinical trials in TB therapy. These clinical trials are fundamental in providing the guidance for enhanced efforts at controlling this ancient and devastating infectious disease (40).
6. Danel C, Kabran M, Inwoley A, et al. QuantiFERON-TB Gold: performance for ruling out active tuberculosis in HIV-infected adults with high CD4 count in Cote d'Ivoire, West Africa. PLoS One 2014;9:e107245.

7. Rangaka MX, Wilkinson RJ, Boulle A, et al. Isoniazid plus antiretroviral therapy to prevent tuberculosis: A randomised double-blind, placebo-controlled trial. Lancet 2014;384:682-90.

8. Durovni B, Saraceni V, van den Hof S, et al. Impact of replacing smear microscopy with Xpert MTB/RIF for diagnosing tuberculosis in Brazil: A stepped-wedge cluster-randomized trial. PLoS Med 2014;11:e1001766.

9. Theron G, Zijenah L, Chanda D, et al. Feasibility, accuracy, and clinical effect of point-of-care Xpert MTB/RIF testing for tuberculosis in primary-care settings in Africa: A multicentre, randomised, controlled trial. Lancet 2014;383:424-35.

10. Merle CS, Fielding K, Sow OB, et al. A four-month gatifloxacincontaining regimen for treating tuberculosis. N Engl J Med 2014;371:1588-98. 
11. Jindani A, Harrison TS, Nunn AJ, et al. High-dose rifapentine with moxifloxacin for pulmonary tuberculosis. N Engl J Med 2014:371:1599-608.

12. Dorman SE, Savic RM, Goldberg S, et al. Daily rifapentine for treatment of pulmonary tuberculosis: A randomized, dose-ranging trial. Am J Resp Crit Care Med 2014 December 9 (Epub ahead of print).

13. Gillespie SH, Crook AM, McHugh TD, et al; REMoxTB Consortium. Four-month moxifloxacin-based regimens for drug-sensitive tuberculosis. N Engl J Med 2014;371:1577-87.

14. Diacon AH, Pym A, Grobusch MP, et al. Multidrug-resistant tuberculosis and culture conversion with bedaquiline. N Engl J Med 2014;371:723-32.

15. Odone A, Amadasi S, White RG, Cohen T, Grant AD, Houben RM. The impact of antiretroviral therapy on mortality in HIV positive people during tuberculosis treatment: A systematic review and meta-analysis. PLoS One 2014;9:e112017.

16. Grinsztejn B, Hosseinipour MC, Ribaudo HJ, et al. Effects of early versus delayed initiation of antiretroviral treatment on clinical outcomes of HIV-1 infection: Results from the phase 3 HPTN 052 randomised controlled trial. Lancet Infect Dis 2014;14:281-90.

17. Collins SE, Jean Juste MA, Koenig SP, et al. CD4 deficit and tuberculosis risk persist with delayed antiretroviral therapy: 5-year data from CIPRA HT-001. Int J Tuberc Lung Dis 2015;19:50-7.

18. Marcy O, Laureillard D, Madec Y, et al. Causes and determinants of mortality in HIV-infected adults with tuberculosis: An analysis from the CAMELIA ANRS 1295-CIPRA KH001 randomized trial. Clin Infect Dis 2014;59:435-45.

19. Havlir DV, Kendall MA, Ive P, et al. Timing of antiretroviral therapy for HIV-1 infection and tuberculosis. N Engl J Med 2011;365:1482-91.

20. Blanc FX, Sok T, Laureillard D, et al. Earlier versus later start of antiretroviral therapy in HIV-infected adults with tuberculosis. N Engl J Med 2011;365:1471-81.

21. Abdool Karim SS, Naidoo K, Grobler A, et al. Integration of antiretroviral therapy with tuberculosis treatment. N Engl J Med 2011;365:1492-501.

22. Abdool Karim SS, Naidoo K, Grobler A, et al. Timing of initiation of antiretroviral drugs during tuberculosis therapy. N Engl J Med 2010;362:697-706.

23. Padayatchi N, Abdool Karim SS, Naidoo K, Grobler A, Friedland G. Improved survival in multidrug-resistant tuberculosis patients receiving integrated tuberculosis and antiretroviral treatment in the SAPiT Trial. Int J Tuberc Lung Dis 2014;18:147-54.

24. Mfinanga SG, Kirenga BJ, Chanda DM, et al. Early versus delayed initiation of highly active antiretroviral therapy for HIV-positive adults with newly diagnosed pulmonary tuberculosis (TB-HAART): A prospective, international, randomised, placebo-controlled trial. Lancet Infect Dis 2014;14:563-71.

25. Luetkemeyer AF, Kendall MA, Nyirenda M, et al. Tuberculosis immune reconstitution inflammatory syndrome in A5221 STRIDE: Timing, severity, and implications for HIV-TB programs. J Acquir Immune Defic Syndr 2014;65:423-8.

26. Grinsztejn B, De Castro N, Arnold V, et al. Raltegravir for the treatment of patients co-infected with HIV and tuberculosis (ANRS 12180 Reflate TB): A multicentre, phase 2, non-comparative, open-label, randomised trial. Lancet Infect Dis 2014;14:459-67.

27. Lan NT, Thu NT, Barrail-Tran A, et al. Randomised pharmacokinetic trial of rifabutin with lopinavir/ritonavirantiretroviral therapy in patients with HIV-associated tuberculosis in Vietnam. PLoS One 2014;9:e84866.

28. Naiker S, Connolly C, Wiesner L, et al. Randomized pharmacokinetic evaluation of different rifabutin doses in African HIV-infected tuberculosis patients on lopinavir/ritonavir-based antiretroviral therapy. BMC Pharmacol Toxicol 2014;15:61.

29. Hatherill M, Geldenhuys H, Pienaar B, et al. Safety and reactogenicity of BCG revaccination with isoniazid pretreatment in TST positive adults. Vaccine 2014;32:3982-8.

30. Thacher EG, Cavassini M, Audran R, et al. Safety and immunogenicity of the M72/AS01 candidate tuberculosis vaccine in HIV-infected adults on combination antiretroviral therapy: A phase I/II, randomized trial. AIDS 2014;28:1769-81.

31. Reither K, Katsoulis L, Beattie T, et al. Safety and immunogenicity of $\mathrm{H} 1 / \mathrm{IC} 31(\mathrm{R})$, an adjuvanted TB subunit vaccine, in HIV-infected adults with CD4+ lymphocyte counts greater than 350 cells $/ \mathrm{mm}^{3}$ : A phase II, multi-centre, double-blind, randomized, placebo-controlled trial. PLoS One 2014;9:e114602.

32. Nell AS, D'Lom E, Bouic P, et al. Safety, tolerability, and immunogenicity of the novel antituberculous vaccine RUTI: Randomized, placebo-controlled phase II clinical trial in patients with latent tuberculosis infection. PLoS One 2014;9:e89612.

33. Mayosi BM, Ntsekhe M, Bosch J, et al. Prednisolone and Mycobacterium indicus pranii in tuberculous pericarditis. N Engl J Med 2014;371:1121-30.

34. Tabarsi P, Fahimi F, Heidarzadeh N, et al. The effect of Garcin(R) in preventing anti-TB-induced hepatitis in newly diagnosed tuberculosis patients. Iran J Pharm Res 2014;13(Suppl):169-76.

35. Hatamkhani S, Khalili H, Karimzadeh I, Dashti-Khavidaki S, Abdollahi A, Jafari S. Carnitine for prevention of antituberculosis drug-induced hepatotoxicity: A randomized, clinical trial. J Gastroenterol Hepatol 2014;29:997-1004.

36. Chand KS, Manchanda RK, Mittal R, Batra S, Banavaliker JN, De I. Homeopathic treatment in addition to standard care in multi drug resistant pulmonary tuberculosis: A randomized, double blind, placebo controlled clinical trial. Homeopathy 2014;103:97-107.

37. Jeremiah K, Denti P, Chigutsa E, et al. Nutritional supplementation increases rifampin exposure among tuberculosis patients coinfected with HIV. Antimicrob Agents Chemother 2014;58:3468-74.

38. Yadav RP, Nishikiori N, Satha P, Eang MT, Lubell Y. Cost-effectiveness of a tuberculosis active case finding program targeting household and neighborhood contacts in Cambodia. Am J Trop Med Hyg 2014;90:866-72.

39. Churchyard GJ, Fielding KL, Lewis JJ, et al; Thibela TB Study Team. A trial of mass isoniazid preventive therapy for tuberculosis control. N Engl J Med 2014;370:301-10.

40. Donoghue HD, Spigelman M, Greenblatt CL, et al. Tuberculosis: From prehistory to Robert Koch, as revealed by ancient DNA. Lancet Infect Dis 2004;4:584-92. 


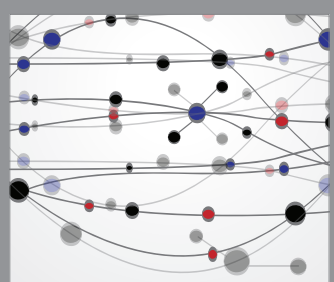

The Scientific World Journal
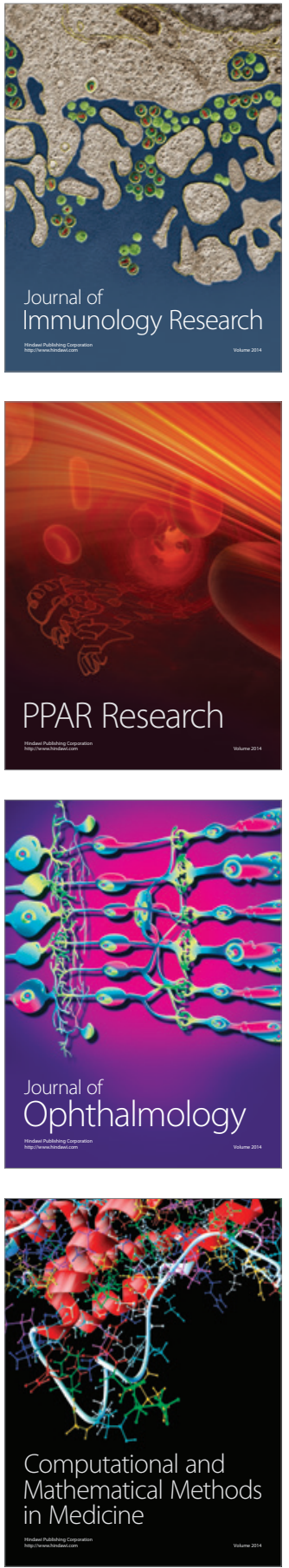

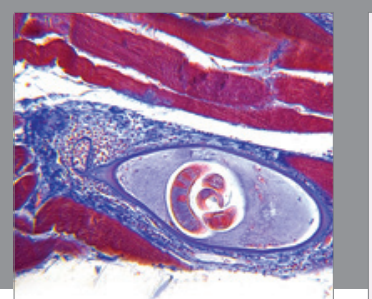

Gastroenterology Research and Practice

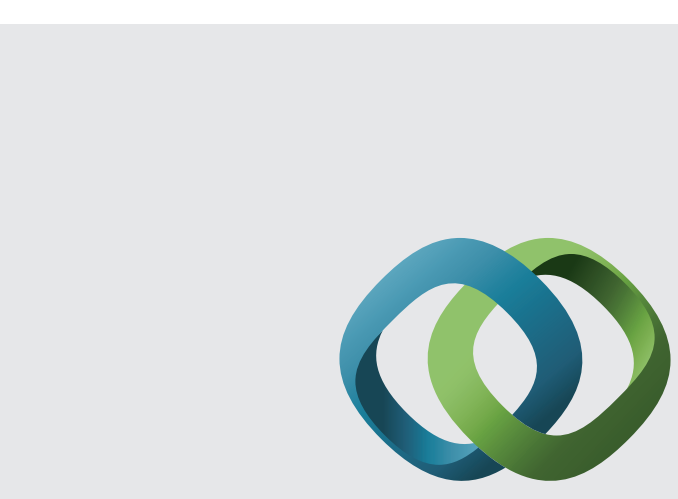

\section{Hindawi}

Submit your manuscripts at

http://www.hindawi.com
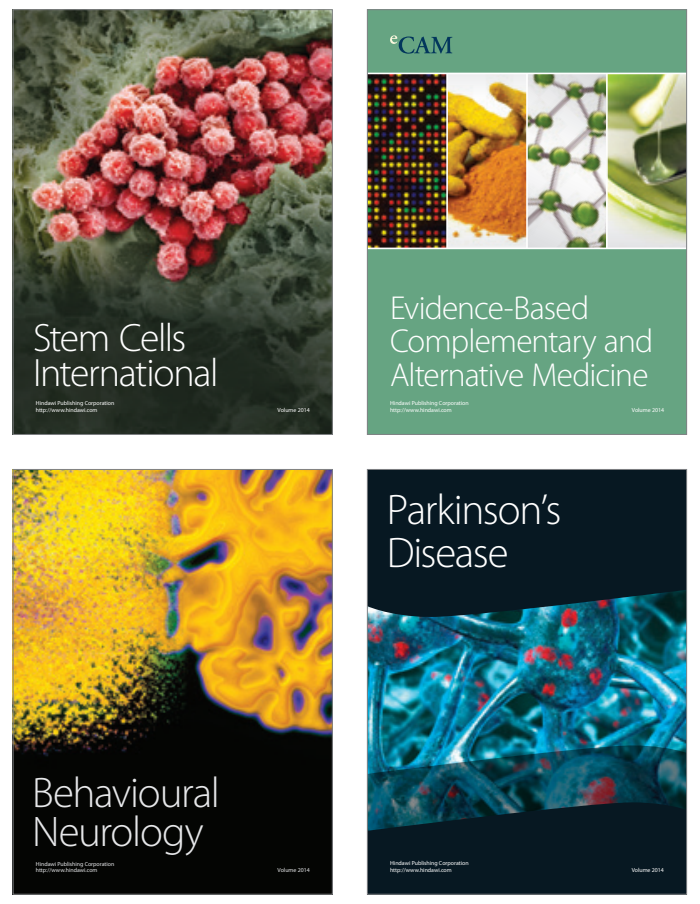
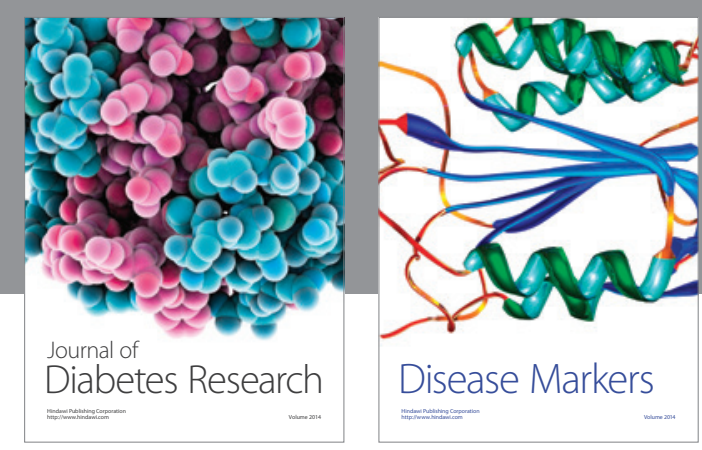

Disease Markers
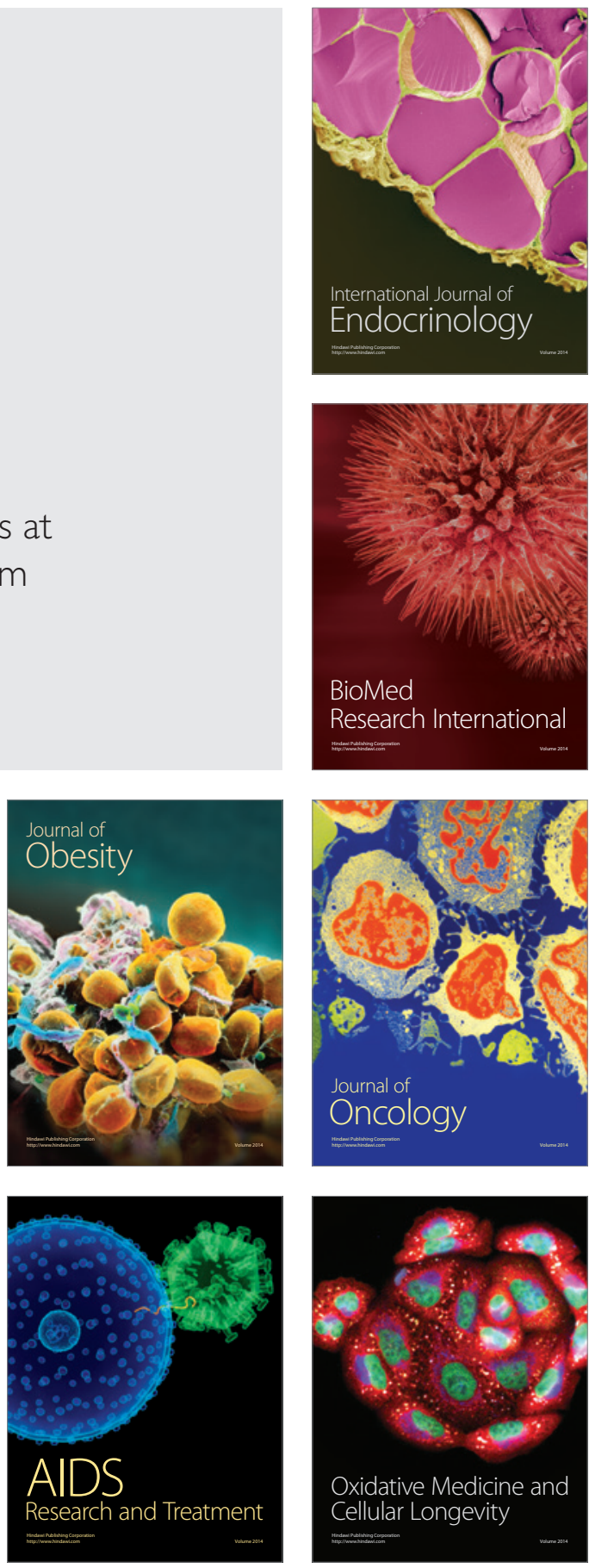